\title{
Cone-Beam Computed Tomography and Radiographs in Dentistry: Aspects Related to Radiation Dose
}

\author{
Diego Coelho Lorenzoni, ${ }^{1}$ Ana Maria Bolognese, ${ }^{1}$ Daniela Gamba Garib, ${ }^{2}$ \\ Fabio Ribeiro Guedes, ${ }^{3}$ and Eduardo Franzotti Sant'Anna ${ }^{1}$ \\ ${ }^{1}$ Department of Orthodontics, Federal University of Rio de Janeiro Dental School, Avenida Professor Rodolpho de Paulo Rocco, \\ Ilha do Fundão 21941-590, Rio de Janeiro, RJ, Brazil \\ ${ }^{2}$ Department of Orthodontics, Bauru Dental School and Hospital of Rehabilitation of Craniofacial Anomalies, Universtity of São Paulo, \\ 17012-101 Bauru, SP, Brazil \\ ${ }^{3}$ Department of Dental Radiology, Federal University of Rio de Janeiro Dental School, 21941-590 Rio de Janeiro, RJ, Brazil
}

Correspondence should be addressed to Eduardo Franzotti Sant’Anna, eduardo.franzotti@gmail.com

Received 14 July 2011; Revised 19 September 2011; Accepted 31 January 2012

Academic Editor: Neil S. Norton

Copyright (c) 2012 Diego Coelho Lorenzoni et al. This is an open access article distributed under the Creative Commons Attribution License, which permits unrestricted use, distribution, and reproduction in any medium, provided the original work is properly cited.

\begin{abstract}
Introduction. The aim of this study was to discuss the radiation doses associated with plain radiographs, cone-beam computed tomography (CBCT), and conventional computed tomography (CT) in dentistry, with a special focus on orthodontics. Methods. A systematic search for articles was realized by MEDLINE from 1997-March 2011. Results. Twenty-seven articles met the established criteria. The data of these papers were grouped in a table and discussed. Conclusions. Increases in kV, mA, exposure time, and field of view (FOV) increase the radiation dose. The dose for CT is greater than other modalities. When the full-mouth series (FMX) is performed with round collimation, the orthodontic radiographs transmit higher dose than most of the large FOV CBCT, but it can be reduced if used rectangular collimation, showing lower effective dose than large FOV CBCT. Despite the image quality, the CBCT does not replace the FMX. In addition to the radiation dose, image quality and diagnostic needs should be strongly taken into account.
\end{abstract}

\section{Introduction}

The high prevalence and increase in the number of children receiving orthodontic care [1] bring up an important issue: the use of ionizing radiation for diagnosis also increases the potential impact on public health [2]. These concerns exist because of the ability of X-rays to induce mutations in DNA, thereby increasing the risk of cancer [3]. Moreover, children may express increased susceptibility to environmental hazards, chronic infection and inflammation, dietary factors, and long-term medication due to differences in the uptake, metabolism, and excretion of potential mutagens [4] and a recent study has suggested a relationship between exposure to dental radiographs and a greater risk of thyroid cancer [5].

During the last century, dental diagnostic imaging was dominated by radiographs, which are two-dimensional representations of three-dimensional structures, with associated overlap and distortion. With the introduction of cone-beam computed tomography ( $\mathrm{CBCT}$ ), there was much interest in the technology due to its advantages: improved image quality, three-dimensional reconstruction, a 1:1 ratio that allowed reliable measurements, the possibility for craniofacial visualization, and lower radiation doses compared to traditional CT.

However, it is necessary to monitor the radiation doses involved in these exams. Some concepts are relevant for this understanding, such as the methodology employed in research studies within the field. The majority of these studies use human head and neck phantoms built with tissues that mimic human tissues in regard to layers and radiation absorption. In some models, human skeletons are used [6]. The phantom is made in the form of detachable cross-sections with apertures created for the placement of dosimeters in the regions of interest. Many of these locations 
would be unfeasible in vivo. The dosimeters measure the absorbed dose in each region/tissue.

The description of the radiation dose transmitted to the patient must be based on the effective dose $(E)$, measured in Sieverts (Sv). This description is recommended by the International Commission on Radiological Protection (ICRP) [7] because it considers not only the dose, but also the type, quantity, sensitivity, and carcinogenic potential of the irradiated tissue [8]. Current estimates of per capita annual U.S. dose are $6200 \mu \mathrm{Sv}$ with almost $3000 \mu \mathrm{Sv}$ coming from diagnostic procedures. Ubiquitous background sources account for $3100 \mu \mathrm{Sv}$ annual dose or $8.5 \mu \mathrm{Sv}$ per day [9].

The effective dose in a given tissue $\left(E_{T}\right)$ is calculated by the following equation [10]: $E_{T}=w_{T} \cdot H_{T}$, where $w_{T}$ is the tissue weighting factor, which represents the radiosensitivity of the tissue/organ and thereby the contribution of this tissue to the total risk, and $H_{T}$ is the equivalent dose for each tissue/organ. The sum $\left(\sum\right)$ of the $E_{T}$ for each tissue/organ provides the total effective dose $(E)$.

The equivalent dose $\left(H_{T}\right)$ for a tissue/organ, in Sv, is represented by the following formula: $H_{T}=w_{R} \cdot D_{T} \cdot f_{T}$, where $w_{R}$ is the radiation weighting factor (for X-rays, this value is $1), D_{T}$ is the mean dose absorbed in the dosimeters in gray (Gy), and $f_{T}$ is the irradiated fraction of tissue in relation to its total volume in the body (normal values described in the literature) [11].

The tissue/organ weighting factors, $\mathrm{w}_{T}$, are provided and updated by the ICRP (Table 1 ). The most widely used version is from 1990 [7] and is based on mortality rates used to estimate the risk of cancers in various tissues. Updates in 2005 [12] and 2007 [10] included the salivary glands and changes in some tissue-weighting factors according to recent rates of cancer incidence, which are better descriptors of cancer burden, especially for those cancer types with high survival rates [13]. The recommendations from 2005 were the draft for the ICRP 2007 recommendations, and the two are, therefore, relatively comparable. Thus, depending on the version of the ICRP recommendations, different effective doses are found for the same level of irradiation. Some articles use the absorbed dose (Gy), which is less relevant because it does not consider the relative contribution of different organs/tissues to the total risk [14].

When using ionizing radiation, the ALARA [15] (as low as reasonably achievable) principle must be respected. Nevertheless, discussions about radiation doses and their contributing factors do exist, and this requires vigilance in obtaining the best possible cost-benefit relationship between dosage and information. Consequently, the sources of radiation used in dentistry (radiography, CBCT, and CT) and the influence of the image acquisition protocol on these doses is discussed, especially in orthodontics.

\section{Materials and Methods}

2.1. Literature Search Strategy. The literature on radiation doses used in dentistry was systematically reviewed. The articles were located by an online search using MEDLINE from 1997 to March 2011. The keyword used in this search was "radiation dose," combined with 31 descriptors to restrict it to dentistry (Figure 1). The bibliographies of the selected articles were analyzed in search of research that was not found on MEDLINE.

2.2. Inclusion Criteria for Articles. Initially, articles in English were selected according to their title and abstract, followed by a complete reading of the text. The studies included in the analysis fulfilled the following criteria:

(1) evaluation of radiation dose in radiographs and/or CBCT and/or CTs used in dentistry;

(2) the use of phantom or thermoluminescent dosimeters;

(3) results that showed effective dose and ICRP used;

(4) tomography of the maxilla and/or mandible and/or the entire head with the assessments of smaller areas discarded;

(5) radiographs included, including a complete periapical examination, and/or a complete interproximal examination, and/or a panoramic and/or lateral cephalometric/PA and/or maxillary/mandibular occlusal examination.

The CBCT studies were divided according to their FOV [11]: small FOV (spherical diameter or cylinder height $\leq 10 \mathrm{~cm}$; captures most of one or both arches, but not all of the anatomy of the maxilla); medium FOV (spherical diameter or cylinder height between 10 and $15 \mathrm{~cm}$; captures the entire dentition and temporomandibular joints, but generally does not include the complete soft profile of the chin and nose, which is necessary for orthodontic care); large or extended FOV (spherical diameter or cylinder height $>15 \mathrm{~cm}$; captures the maxillofacial complex, chin and nose).

\section{Results}

There were 94.742 articles identified with the keyword radiation dose, which were reduced to 27 after application of the criteria. Table 2 lists these data. It is important to know that some of the devices presented in Table 2 are not the most current versions available. For example, the CBCT devices such as Classic i-CAT, NewTom 9000, NewTom 3G, and Iluma already have new versions (Next Generation iCAT, NewYom 5G and Iluma Elite). The CB MercuRay is not currently available for purchase. They were all kept in Table 2 because they can still be used in some centers.

\section{Discussion}

Methodological variations explain the different doses for the same exam, where these include phantoms made by different companies or positioned asymmetrically, as well as variations in dosimeters, their sensors [16], their locations on the phantoms, and their number [17]. Many researchers do not include the calvaria $[6,8,15,18-24]$ and cervical vertebrae $[18,21,23,24]$ when counting the red bone marrow, esophagus $[8,18-21,23-26]$, skin [25], and remaining 


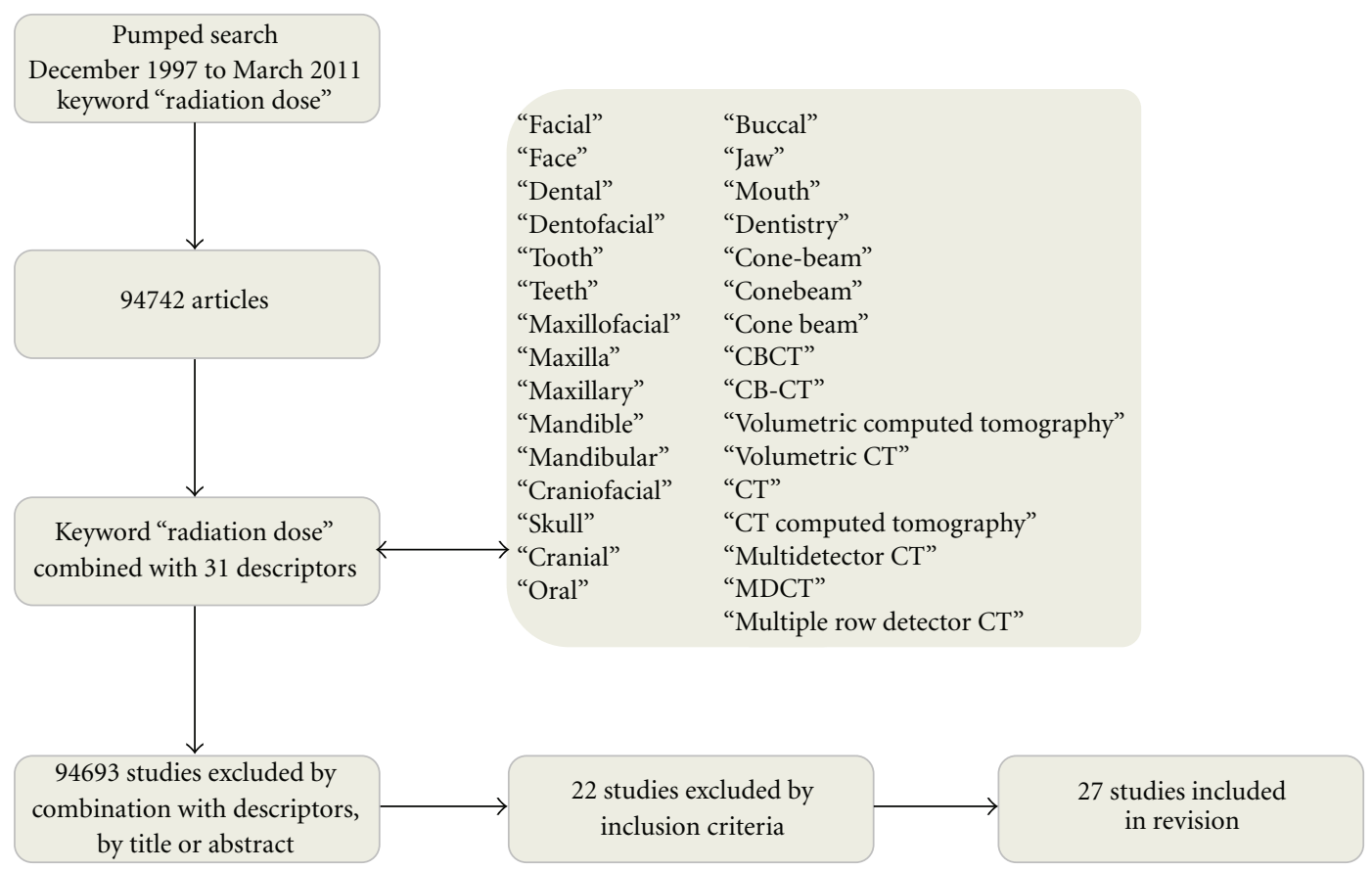

FIGURE 1: Flow chart of the search process.

TABLE 1: Tissue-weighting factors for calculation of effective radiation dose.

\begin{tabular}{lccc}
\hline Tissue & ICRP 1990 & ICRP 2005 & ICRP 2007 \\
\hline Bone Marrow & 0.12 & 0.12 & 0.12 \\
Breast & 0.05 & 0.12 & 0.12 \\
Colon & 0.12 & 0.12 & 0.12 \\
Lung & 0.12 & 0.12 & 0.12 \\
Stomach & 0.12 & 0.05 & 0.12 \\
Gonads & 0.20 & 0.05 & 0.08 \\
Esophagus & 0.05 & 0.05 & 0.04 \\
Bladder & 0.05 & 0.05 & 0.04 \\
Liver & 0.05 & 0.05 & 0.04 \\
Thyroid & 0.05 & 0.01 & 0.04 \\
Bone surface & 0.01 & 0.01 & 0.01 \\
Brain & RT & 0.01 & 0.01 \\
Skin & 0.01 & 0.01 & 0.01 \\
Salivary glands & Not included & 0.01 & 0.01 \\
Kidney & RT & $0.10^{\mathrm{b}}$ & RT \\
Remainder Tissues & $0.05^{\mathrm{a}}$ & $0.12^{\mathrm{c}}$ \\
\hline
\end{tabular}

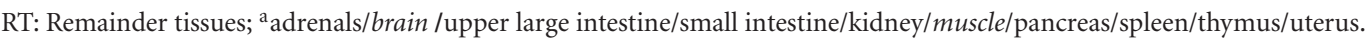

${ }^{\mathrm{b}}$ Adipose tissue/adrenals/connective tissue/extrathoracic airways/gallbladder/heart wall/lymphatic nodes/muscle/pancreas/prostate/spleen/thymus/uterus/cervix.

${ }^{c}$ Adrenals/extrathoracic region/gallbladder/heart/prostate/kidneys/small intestine/lymphatic nodes/oral mucosa/muscle/pancreas/spleen/thymus/uterus/cervix (text in boldface represents tissues used for calculation of maxillofacial dose).

tissues in the calculation of the effective dose $[6,18,19$, $21,23,24]$. The ICRP version used is important due to the inherent variations in the different weighting factors. The 1990 ICRP [7] did not include the salivary glands, which are highly irradiated in dentistry, and some authors included them among the remainder tissues of the ICRP, which considerably increased the effective dose (Table 2).
This tissue was incorporated in the ICRP from 2005 [12] and 2007 [10], and this explains the larger doses measured.

4.1. Image Acquisition Protocol. Increases in $\mathrm{kV}, \mathrm{mA}$, and exposure time result in higher effective doses for any exam $[6,11,12,16,27-29]$. The adjustments in CBCT images vary; 
Table 2: Effective doses. (ExcGland or IncGland: salivary glands excluded or included; Mx: Maxilla; Md: Mandible).

\begin{tabular}{|c|c|c|c|c|}
\hline \multirow{3}{*}{ Exams/equipment/adjustment provided } & \multicolumn{4}{|c|}{ Effective Dose $(\mu \mathrm{Sv})$} \\
\hline & \multicolumn{2}{|c|}{ ICRP $60-1990$} & \multirow{2}{*}{ ICRP 2005} & \multirow{2}{*}{ ICRP 103-2007 } \\
\hline & ExcGland & IncGland & & \\
\hline \multicolumn{5}{|l|}{ PANORAMIC RADIOGRAPHS } \\
\hline PM2002CCProlinePlanmeca/70 kVp/7 mA/18 s [25] & 3.8 & & & \\
\hline VeraviewepocsMorita $77 \mathrm{kV} / 5 \mathrm{~mA} / 8.1 \mathrm{~s}$ [8] & & & 5.2 & \\
\hline OrthophosSiemens/62 kV/16 mA/14.1s [38] & 9 & 16.4 & & \\
\hline PM2002CCProLinePlanmeca/64 kV/6 mA/15 s [39] & 4 & 9 & & \\
\hline PromaxPlanmeca/66 kV/6 mA/16s [16] & 17 & 26 & & \\
\hline PM2002CCProlinePlanmeca/73 kV/5 mA/15 s [18] & & 10 & & \\
\hline Digital/PM2002CCProline2000Planmeca/66 kV/4 mA/18 s [16] & 8 & 12 & & \\
\hline Digital/PM2002CCProline2000Planmeca/66 kV/8 mA/18 s [16] & 23 & 38 & & \\
\hline Digital/CranexExcelSoredex/65 kV/6 mA/19s [40] & 4.5 & 12.3 & & \\
\hline Digital/Verawiewepocs5DMorita/70 kV/4 mA/8.2 s [40] & 2.5 & 5.5 & & \\
\hline Digital/ECProlinePlanmeca/64 kV/7 mA/18.3 s [40] & 5.7 & 14.9 & & \\
\hline Digital/Orthoralix9200DDEGendex/74 kV/4 mA/12 s [40] & 2.4 & 4.7 & & \\
\hline Digital/ProMaxPlanmeca/Adult [6] & 20 & & 23 & \\
\hline Digital/ProMaxPlanmeca/68 kV/13 mA/16 s [13] & 7.1 & & & 24.3 \\
\hline Digital/OrthophosXGSirona/64 kV/8 mA/14.1 s [13] & 4.3 & & & 14.2 \\
\hline Digital/OrthophosPlusDSSirona/66 kVp/16 mA/14.1 s [30] & 6.2 & 22 & & \\
\hline Digital/VeraviewepocsMorita/67 kV/5 mA/8.1 s [8] & & & 2.7 & \\
\hline Digital/Veraviewepocs3DMorita/70 kV/5 mA/7.4 s [8] & & & 2.9 & \\
\hline Digital/CranexTomeSoredex/70 kV/4 mA/15 s [40] & 3.3 & 8.1 & & \\
\hline \multicolumn{5}{|l|}{ LATERAL CEPHALOMETRIC RADIOGRAPHS } \\
\hline OrthophosCSiemens/77 kV/14 mA/0.5 s [19] & 2.3 & & & \\
\hline PM2002CCProLinePlanmeca/70 kV/12 mA/0.9s [39] & 2 & 3 & & \\
\hline CranexTomeSoredex/70 kVp/10 mA/0.4 s [20] & 3 & 3.7 & & \\
\hline CranexTomeSoredex/Collimation/70 kVp/10 mA/0.4s [20] & 1.6 & 2.2 & & \\
\hline PM2002CCProlinePlanmeca/80 kV/12 mA/0.5 s [18] & & 5 & & \\
\hline Digital/OrthophosDSCephSiemens/73 kV/15 mA/15.8s [19] & 1.1 & & & \\
\hline Digital/ProLineCephCMPlanmeca/Collimation/70 kVp/10 mA/23 s [21] & 1.7 & 3.4 & & \\
\hline Digital/CranexTomeSoredex/Collimation/70 kVp/4 mAs [21] & 1.6 & 2.2 & & \\
\hline Digital/InterayVarian $/ 77 \mathrm{kVp} / 6.5 \mathrm{mAs}[13]$ & 3.7 & & & 5.6 \\
\hline \multicolumn{5}{|l|}{ PA CEPHALOMETRIC RADIOGRAPHS } \\
\hline Digital/InterayVarian/75 kVp/11 mAs [13] & 3.9 & & & 5.1 \\
\hline \multicolumn{5}{|l|}{ INTRAORAL RADIOGRAPHS } \\
\hline $\begin{array}{l}\text { IntraPlanmeca/FullMouthRadiographs/70 kV/8 mA/Digital or F-speed } \\
\text { film/RectangularCollimation [13] }\end{array}$ & 12.2 & & & 34.9 \\
\hline $\begin{array}{l}\text { IntraPlanmeca/FullMouthRadiographs/70 kV/8 mA/Digital or F-speed } \\
\text { film/RoundCollimation [13] }\end{array}$ & 58.4 & & & 170.7 \\
\hline IntraPlanmeca/FullMouthRadiographs/RoundCollimation/Adult [6] & 115 & & 129 & \\
\hline $\begin{array}{l}\text { IntraPlanmeca/Bitewing }(04) / 70 \mathrm{kV} / 8 \mathrm{~mA} / \text { Digital or F-speed } \\
\text { film/RectangularCollimation [6] }\end{array}$ & 1 & & & 5 \\
\hline SiemensHeliodent70Dentotime/OcclusalMx [18] & & 7 & & \\
\hline \multicolumn{5}{|l|}{ LARGE FOV CONE BEAM CT } \\
\hline Classic i-CAT/FOV22 cm/120 kV/3-8 mA [27] & 92.8 & & & 182.1 \\
\hline Classic i-CAT/FOV22 cm/120 kV/5.7 mA [12] & 134.8 & & 193.4 & \\
\hline Classic i-CAT/FOV22 cm/120 kV/3-8 mA/2 × $20 \mathrm{~s}$ [28] & & & & 82 \\
\hline Next Generation i-CAT/FOV23 cm/120 kV/5 mA/19 mAs/8.9 s [11] & 37 & & & 74 \\
\hline NewTom3G/FOV19 cm/110 kV/1.5 mA/8.09 mAs/36 s [11, 12] & 44.7 & & 58.9 & 68 \\
\hline
\end{tabular}


Table 2: Continued.

\begin{tabular}{|c|c|c|c|c|}
\hline \multirow{3}{*}{ Exams/equipment/adjustment provided } & \multicolumn{4}{|c|}{ Effective Dose $(\mu \mathrm{Sv})$} \\
\hline & \multicolumn{2}{|c|}{ ICRP 60-1990 } & \multirow{2}{*}{ ICRP 2005} & \multirow{2}{*}{ ICRP 103-2007 } \\
\hline & ExcGland & IncGland & & \\
\hline NewTom3G/FOV19 cm/110 kV/<15 mA [28] & & & & 30 \\
\hline NewTom9000/FOV23 cm/110 kV/5.4 mA [15] & & & 56.2 & \\
\hline CBMercuRay/FOV19 cm/100 kV/10 mA/100 mAs/10 s [11, 12] & 476.6 & & 557.6 & 569 \\
\hline CBMercuRay/FOV19 cm/120 kV/15 mA/150 mAs/10 s [11, 12] & 846.9 & & 1025.4 & 1073 \\
\hline CBMercuRay/FOV19 cm/100 kV/15 mA [6] & 415 & & 479 & \\
\hline CBMercuRay/FOV19 cm/120 kV/15 mA [6] & 656 & & 761 & \\
\hline CBMercuRay/FOV19 cm/100 kV/10 mA [6] & 264 & & 306 & \\
\hline CBMercuRay/FOV19 cm/100 kV/5 mA [6] & 153 & & 177 & \\
\hline CBMercuRay/FOV19 cm/100 kV/2 mA [6] & 75 & & 86 & \\
\hline Iluma/FOV19 cm/120 kV/1 mA/20 mAs/20 s [11] & 50 & & & 98 \\
\hline Iluma/FOV19 cm/120 kV/3.8 mA/152 mAs/40 s [11] & 252 & & & 498 \\
\hline Kodak9500/FOV18 cm/80 kV/86.4 mAs [29] & 52 & & & 93 \\
\hline Kodak9500/FOV18 cm/85 kV/108 mAs [29] & 92 & & & 163 \\
\hline Kodak9500/FOV18 cm/90 kV/108 mAs [29] & 148 & & & 260 \\
\hline Kodak9500/FOV18 cm/90 kV/108 mAs [17] & & & & 136 \\
\hline SkyView/FOV17 cm/90 kV/51 mAs [17] & & & & 87 \\
\hline \multicolumn{5}{|l|}{ MEDIUM FOV CONE BEAM CT } \\
\hline Classic i-CAT/FOV13 cm/120 kV/3-8 mA [27] & 39.5 & & & 110.5 \\
\hline Classic i-CAT/FOV13 cm/120 kV/5.7 mA [12] & 68.7 & & 104.5 & \\
\hline Classic i-CAT/FOV13 cm/120 kV/23.87 mA [15] & & & 61.1 & \\
\hline Classic i-CAT/FOV13 cm/120 kV/3-8 mA/10 s [28] & & & & 48 \\
\hline Classic i-CAT/FOV13 cm/120 kV/3-8 mA/40 s [28] & & & & 77 \\
\hline Classic i-CAT/FOV13 cm/120 kV/5 mA/19 mAs/20 s [11] & 29 & & & 69 \\
\hline Next Generation i-CAT/FOV13 cm/120 kV/5 mA/19 mAs/8.9s [11] & 36 & & & 87 \\
\hline Next Generation i-CAT/FOV13 cm/120 kV/18.5 mAs [17] & & & & 83 \\
\hline NewTom9000/FOV13 cm/110 kV/3.2 mA [30] & 36.9 & 77.9 & & \\
\hline NewTom9000/FOV13 cm/110 kV/3.5 mA/18 s [26] & 50.3 & & & \\
\hline NewTom9000/FOV13 cm/110 kV/3.4 mA/17 s [22] & 35 & 64 & & \\
\hline NewTom9000/FOV13 cm/110 kV/3.4 mA/17 s/Thyroid Protector [22] & 23 & 52 & & \\
\hline NewTom3G/FOV $15 \mathrm{~cm} / 110 \mathrm{kV} /<15 \mathrm{~mA}[28]$ & & & & 57 \\
\hline NewTom5Gi/FOV15 cm/110 kV/8.8 mAs [17] & & & & 194 \\
\hline CBMercuRay/FOV15 cm/120 kV/15 mA/120/mAs/10 s [11] & 288.9 & & 435.5 & 560 \\
\hline CBMercuRay/FOV15 cm/100 kV/15 mA [6] & 354 & & 402 & \\
\hline CBMercuRay/FOV15 cm/120 kV/15 mA [6] & 601 & & 680 & \\
\hline Galileos/FOV15 cm/85 kV/5 mA/21 mAs/14 s [11] & 28 & & & 70 \\
\hline Galileos/FOV15 cm/85 kV/7 mA/42 mAs/14 s [11] & 52 & & & 128 \\
\hline GalileosComfort/FOV15 cm/85 kV/28 mAs [17] & & & & 84 \\
\hline Kodak9500/FOV15 cm/80 kV/86.4 mAs [29] & 39 & & & 76 \\
\hline Kodak9500/FOV15 cm/85 kV/108 mAs [29] & 49 & & & 98 \\
\hline Kodak9500/FOV15 cm/90 kv/108 mAs [29] & 76 & & & 166 \\
\hline IlumaElite/FOV14 cm/120 kV/76 mAs [17] & & & & 368 \\
\hline Scanora3D/FOV13.5 cm/85 kV/48 mAs [17] & & & & 68 \\
\hline \multicolumn{5}{|l|}{ SMALL FOV CONE BEAM CT } \\
\hline Classic i-CAT/FOV6 cmMx/120 kV/3-8 mA [27] & 9.7 & & & 36.5 \\
\hline Classic i-CAT/FOV6 cmMx/120 kV/3-8 mA/HighResolution [27] & 18.5 & & & 68.3 \\
\hline Classic i-CAT/FOV6 cmMx/120 kV/3-8 mA/20 s [28] & & & & 45 \\
\hline Classic i-CAT/FOV6 cmMx/120 kV/3-8 mA/40 s [28] & & & & 77 \\
\hline
\end{tabular}


TABle 2: Continued.

\begin{tabular}{|c|c|c|c|c|}
\hline \multirow{3}{*}{ Exams/equipment/adjustment provided } & \multicolumn{4}{|c|}{ Effective Dose $(\mu \mathrm{Sv})$} \\
\hline & \multicolumn{2}{|c|}{ ICRP 60-1990 } & \multirow{2}{*}{ ICRP 2005} & \multirow{2}{*}{ ICRP 103-2007 } \\
\hline & ExcGland & IncGland & & \\
\hline Classic i-CAT/FOV6 cmMd/120 kV/3-8 mA [27] & 23.9 & & & 75.3 \\
\hline Classic i-CAT/FOV6 cmMd/120 kV/3-8 mA/HighResolution [27] & 47.2 & & & 148.5 \\
\hline Classic i-CAT/FOV6 cmMd/120 kV/3-8 mA/20 s [28] & & & & 34 \\
\hline Classic i-CAT/FOV6 cmMd/120 kV/3-8 mA/40 s [28] & & & & 64 \\
\hline Classic i-CAT/FOV8 cm/120 kV/3-8 mA/40 s [28] & & & & 37 \\
\hline Next Generation i-CAT/FOV6 cmMd/120 kV/18.5 mAs [17] & & & & 45 \\
\hline NewTom9000/FOVMx [30] & 19.9 & 41.5 & & \\
\hline NewTom9000/FOVMd [30] & 34.7 & 74.7 & & \\
\hline NewTom5G/FOV10 cm/110 kV/10.4 mAs [17] & & & & 83 \\
\hline NewTom5Gi/FOV8 cm/110 kV/43 mAs [17] & & & & 265 \\
\hline CBMercuRay/FOV10 cmMx/120 kV/15 mA/150 mAs/10s [11, 12] & 168.4 & & 283.3 & 407 \\
\hline CBMercuRay/FOV10 cm/100 kV/15 mA [6] & 328 & & 369 & \\
\hline CBMercuRay/FOV10 cm/120 kV/15 mA [6] & 535 & & 603 & \\
\hline CBMercuRay/FOV10 cm/120 kV/15 mA [23] & 451.8 & & & 510.5 \\
\hline Promax3D/FOV8 cm/84kVp/12 mA/6s [41] & 269 & & & 674 \\
\hline Promax3D/FOV8 cm/84 kV/12 mA/72 mAs/18 s [11] & 151 & & & 488 \\
\hline Promax3D/FOV8 cm/84 kV/16 mA/96 mAs/18 s [11] & 203 & & & 652 \\
\hline Promax3D/FOV8 cm/84 kV/8 mA/12 s/NormalResolution [42] & & & & 102 \\
\hline Promax3D/FOV8 cm/84 kV/10 mA/12 s/NormalResolution [42] & & & & 169 \\
\hline Promax3D/FOV8 cm/84 kV/12 mA/12 s/NormalResolution [42] & & & & 216 \\
\hline Promax3D/FOV8 cm/84 kV/14 mA/12 s/NormalResolution [42] & & & & 272 \\
\hline Promax3D/FOV8 cm/84 kV/16 mA/12 s/NormalResolution [42] & & & & 298 \\
\hline Promax3D/FOV8 cm/84 kV/8 mA/2.8 s/LowDose [42] & & & & 30 \\
\hline Promax3D/FOV8 cm/84 kV/16 mA/12 s/HighDose [42] & & & & 306 \\
\hline Promax3D/FOV8 cm/84 kV/8 mA/8.3 s/LowDose [42] & & & & 87 \\
\hline Promax3D/FOV8 cm/84 kV/169 mAs/HighDose [17] & & & & 122 \\
\hline Promax3D/FOV8 cm/84 kV/19.9 mAs/LowDose [17] & & & & 28 \\
\hline PreXion3D/FOV8.1 cm/90 kV/4 mA/76 mAs/19s [11] & 66 & & & 189 \\
\hline PreXion3D/FOV8.1 cm/90 kV/4 mA/148 mAs/37 s [11] & 154 & & & 388 \\
\hline 3D Accuitomo 170/FOV5 cmMx/90 kV/87.5 mAs [17] & & & & 54 \\
\hline Kodak9500/FOV8 cm/90 kV/108 mAs [17] & & & & 92 \\
\hline PicassoTrio HighDose/FOV7 cm/85 kV/127 mAs [17] & & & & 123 \\
\hline PicassoTrio LowDose/FOV7 cm/85 kV/91 mAs [17] & & & & 81 \\
\hline Scanora 3D/FOV7.5 cmMx/85 kV/30 mAs [17] & & & & 46 \\
\hline Scanora 3D/FOV7.5 cmMd/85 kV/30 mAs [17] & & & & 47 \\
\hline Scanora 3D/FOV7.5 cmMxMd/85 kV/30 mAs [17] & & & & 45 \\
\hline Veraviewpocs3D/FOV8 cm/70 kV/51 mAs [17] & & & & 73 \\
\hline \multicolumn{5}{|l|}{ CONVENTIONAL CT } \\
\hline $\begin{array}{l}\text { SomatomVolumeZoom4/Scan } 22.6 \mathrm{cmFullHead} / 120 \mathrm{kV} / \\
90 \mathrm{~mA} / 44.12 \mathrm{~s} / \text { Slice } 0.75 \mathrm{~mm}[28]\end{array}$ & & & & 1110 \\
\hline $\begin{array}{l}\text { SomatomSensation } 16 / \mathrm{Scan} 22.5 \mathrm{cmFullHeadl} / 120 \mathrm{kV} / 90 \mathrm{~mA} / \\
29.48 \mathrm{~s} / \mathrm{slice} 0.75 \mathrm{~mm}[28]\end{array}$ & & & & 995 \\
\hline Mx8000IDTPhilips/Scan22.5 cmFullHead/120 kV/140 mA/29.6 s/Slice0.75 mm [28] & & & & 1160 \\
\hline Somatom64/Scan $12 \mathrm{~cm} / 120 \mathrm{kV} / 90 \mathrm{~mA}[11]$ & 453 & & & 860 \\
\hline Somatom64CareDose4D/Scan $12 \mathrm{~cm} / 120 \mathrm{kV} / 90 \mathrm{~mA}[11]$ & 285 & & & 534 \\
\hline $\begin{array}{l}\text { SomatomPlusVolumeZoom4/ScanMx+Md/Slice } 1.25 \mathrm{~mm} / 21.25 \mathrm{~s} / 120 \mathrm{kVp} / \\
150 \mathrm{~mA}[18]\end{array}$ & & 2110 & & \\
\hline
\end{tabular}


TABle 2: Continued.

\begin{tabular}{|c|c|c|c|c|}
\hline \multirow{3}{*}{ Exams/equipment/adjustment provided } & \multicolumn{4}{|c|}{ Effective Dose $(\mu \mathrm{Sv})$} \\
\hline & \multicolumn{2}{|c|}{ ICRP 60-1990 } & \multirow{2}{*}{ ICRP 2005} & \multirow{2}{*}{ ICRP 103-2007 } \\
\hline & ExcGland & IncGland & & \\
\hline SomatomSensation/Scan $10 \mathrm{~cm} / 120 \mathrm{kV} / 90 \mathrm{~mA}$ [15] & & & 429.7 & \\
\hline ExcelTwin/Scan9.6 cm/120 kV/300 mAs/Slice2 mm/2sporslice [39] & 314 & 924 & & \\
\hline HiSpeedQX/i/Scan7.7 cmMx+Md/120 kV/100 mA [23] & 595.6 & & & 768.9 \\
\hline SomatomVolumeZoom4/Scan7.2 cmMd/120 kV/90 mA/15.16s/ Slice0.75 mm [28] & & & & 494 \\
\hline SomatomSensation16/Scan6.3 cmMd/120 kV/90 mA/7.87 s/Slice0.75 mm [28] & & & & 474 \\
\hline Mx8000IDTPhilips/Scan6 cmMd/120 kV/140 mA/7.89 s/ Slice0.75 mm [28] & & & & 541 \\
\hline SomatomPlus4VolumeZoom Scan5.2 cmMd/120 kV/100 mAs [24] & & & 250 & \\
\hline ElscintExcel2400/ScanMd/120 kVp/315 mAs [43] & 2426 & 3324 & & \\
\hline SomatomPlus4VolumeZoom/ScanMd/Slice1.25 mm/12.64 s/ $120 \mathrm{kVp} / 150 \mathrm{~mA}$ [18] & & 1320 & & \\
\hline SomatomPlusVolumeZoom4/ScanMx/Slice 1.25 mm/9.47 s/ $120 \mathrm{kVp} / 150 \mathrm{~mA}$ [18] & & 1400 & & \\
\hline ElscintExcel2400/ScanMx/120 kVp/315 mAs [43] & 1031 & 1202 & & \\
\hline
\end{tabular}

for the i-CAT, the $\mathrm{kV}, \mathrm{mA}$, and exposure time are established by the manufacturer and do not vary from patient to patient. That is, the same dose is used for patients of different sizes and different ages. In children, this may be higher than needed for a diagnosis. For the NewTom 3G, exposure is also set by the manufacturer, but a dynamic process identifies the radiation needed, and the $\mathrm{mA}$ is adjusted during the exposure. For the CB MercuRay, the operator defines $\mathrm{kV}$ and $\mathrm{mA}$. Inexperienced operators tend to increase $\mathrm{kV}$ and $\mathrm{mA}$ because the overexposed images appear to be adequate with reduced noise, which increases the risk of overexposure [12].

For CBCT, smaller FOV normally generates lower radiation doses, similar to the action of collimators $[6,12,17,27-$ 29]. In general, the mandibular FOV has a larger dose than the maxillary $[27,30]$, because the salivary glands, thyroid, and esophagus are more irradiated in this exam. The chosen FOV must be the smallest that will encompass the region of interest [6]. For example, the medium FOV $(13 \mathrm{~cm})$ from the NewTom/i-CAT is often enough to reach the regions required in many children for orthodontics. With the large FOV, unnecessary areas are irradiated in these "minor" children, increasing the effective dose. On the other hand, the large FOV is always necessary in adults. The operator is responsible for choosing the appropriate FOV, large or medium, according to the size of the child.

4.2. $C B C T$ versus $C T$. The effective dose generated by $\mathrm{CT}$ is generally higher than that of CBCT. When analyzing the dose according the 2007 ICRP, the head CT requires doses between 995 and $1160 \mu \mathrm{Sv}$, whereas the large FOV CBCT requires 30 to $68 \mu \mathrm{Sv}$ for the NewTom 3G, $74 \mu \mathrm{Sv}$ for the Next Generation i-CAT, 82 to $182.1 \mu \mathrm{Sv}$ for the Classic i-CAT, $87 \mu \mathrm{Sv}$ for the SkyView, 93 to $260 \mu \mathrm{Sv}$ for the Kodak 9500, and 98 to $498 \mu \mathrm{Sv}$ for the Iluma. The CB MercuRay approaches the radiation levels of standard CT, with doses between 569 and $1073 \mu \mathrm{Sv}$. High doses are observed for CT even when areas are reduced, ranging between 534 and $860 \mu \mathrm{Sv}$ for the maxilla and mandible. This represents a higher dose emitted by CT, especially in relation to the NewTom $3 \mathrm{G}$ and i-CAT CBCT devices. The CT dose is also high in relation to radiographs, which emit doses of 14.2 to $24.3 \mu \mathrm{Sv}$ for the panoramic radiograph, $5.4 \mu \mathrm{Sv}$ for the lateral cephalometric radiograph and 34.9 to $170.7 \mu \mathrm{Sv}$ for a complete intraoral examination.

4.3. CBCT versus Conventional Radiographs. In this transition phase of image diagnosis, a question frequently arises: "to how many radiographs is the radiation dose of CBCT equivalent?" Despite the straightforward nature of the question, the answer involves many nuances.

The characteristics of an intraoral radiograph influence its effective dose, such as film sensitivity (when not digital) and, especially, the type of collimation (rectangular or circular). Intraoral radiographs with circular collimation and films that are not sensitive (D-speed) yielding doses that are much greater than sensitive (E/F-speed) and digital films with rectangular collimation. The dose for the digital/Fspeed complete intraoral exam with rectangular collimation $(34.9 \mu \mathrm{Sv})$ is close to 4.9 times lower than one with circular collimation $(170.7 \mu \mathrm{Sv})$ [13]. The NCRP [31] and the American Dental Association [32] recommend rectangular collimation for periapical and bitewing radiographs, the use of a thyroid protector and the avoidance of using films lower than E-speed (preferably F-speed/digital). In terms of extraoral radiographs, according to ICRP 2005/2007, the doses are between 2.7 and $24.3 \mu \mathrm{Sv}$ for the panoramic and $5.6 \mu \mathrm{Sv}$ for the lateral cephalometric.

Many orthodontists do not request a full-mouth series of intraoral radiographs for orthodontic planning and this practice greatly reduces the dose of radiation imparted to the patient when compared to CBCT exposure. This is particularly important when dealing with young children that are more susceptible to radiation [4]. However, in some instances, it hampers the diagnosis since the panoramic radiograph shows large distortions that prevent the diagnosis of more subtle changes, such as caries and root resorption in early stages. Thus, these radiographs should be taken in 
patients with permanent dentition that will begin full braces orthodontic treatment to search for dental diseases and to serve as a precise record of each teeth and adjacent bone during and posttreatment. Panoramics should also be taken during comprehensive orthodontic treatment to visualize the entire maxilla and mandible including the teeth, maxillary sinuses, nasal cavity, and condyles.

Therefore, in the initial orthodontic radiographic documentation (ORD), which often includes full mouth series of intraoral radiographs (FMX), panoramic, and lateral cephalometric radiographs, the total dose varies between 43.2 and $200.6 \mu \mathrm{Sv}$, depending on the collimation of intraoral radiographs. The large FOV of most CBCT scanners provides lower doses than the ORD with FMX using circular collimation. If rectangular collimation is used, the ORD presents lower effective dose.

It is not enough to compare doses between diagnostic procedures, because diagnostic quality cannot be separated from the dose used. Objective studies of the impact of CBCT image quality on diagnostic performance must be conducted before any definitive conclusions are drawn about the differences generated by reduced doses [12]. Current data describe the reconstructions of lateral teleradiography of CBCT as having similar precision to conventional radiographs [33] in addition to high intra- and interexaminer reproducibility [34]. Comparisons between CBCT images, periapical radiography, and clinical evaluations have not demonstrated significant differences in the extent of periodontal defects, but CBCT allows for the observation of all bone defects and better inspection of craters and furcation defects [35]. However, delicate structures such as the trabecular bone and the periodontal ligament display lower visibility and higher variability between CBCT and CT than do other structures [36]. Conventional radiography has advantages in terms of contrast, the quality of the bone image and delineation of the lamina dura, in addition to superior performance in the evaluation of the periodontal space compared to CBCT [37] and is, therefore, indispensable for accurate periapical diagnosis.

4.4. Differences between CBCT Devices. The CBCT dose varies according to the CBCT device. Among the better known large FOV CBCT, the CB MercuRay provides the greatest radiation, followed by the Classic i-CAT, the Kodak 9500, the Iluma, the Next Generation i-CAT, and the NewTom 3G. Considering the large FOV (ICRP2005) [12], the radiation doses of the Classic i-CAT and the CB MercuRay are 3.3 and 9.5 to 17 times greater, respectively, than that of the NewTom 3G. The Next Generation i-CAT comes close to the NewTom 3G (ICRP 2007) in terms of radiation level because it scans more quickly than the Classic i-CAT.

Considering the large FOV CBCT, a general conclusion, based on values described in Table 2 , is that the effective doses from most devices are found in the 30-200 $\mu$ Sv range. Although the geometry of image acquisition is basically the same, the differences in collimation of the cone beam, as well as the X-ray exposure factors, lead to considerable differences in absorbed dose for all organs in the head and the neck regions. A single effective dose is not a concept that should be used for CBCT when compared to alternative radiographic methods such as panoramic, intraoral radiography, and conventional CT. The range of doses among devices is too large to consider them as a single modality [17].

In addition to controlling the settings of tomographs, radiation levels can vary due to exposure times and radiation beams. The NewTom 3G scans in $36 \mathrm{~s}$ but emits X-ray for only $5.4 \mathrm{~s}$. Similarly, the Classic i-CAT (FOV $13 \mathrm{~cm}$ ) scans in $20 \mathrm{~s}$, but the X-ray tube is only activated for $3.3 \mathrm{~s}$. The large FOV in the i-CAT involves two FOV $13 \mathrm{~cm}$ scans, performed sequentially and interlaced to create a greater volume. Double scanning preserves the quality of FOV $13 \mathrm{~cm}$ but requires almost double the exposure time. The CB MercuRay scans in $11 \mathrm{~s}$ and emits for $10 \mathrm{~s}$. Thus, the exposure for the CB MercuRay is continuous, whereas for the NewTom $3 \mathrm{G}$ and the $\mathrm{i}$-CAT it is pulsed; consequently, the latter two use radiation more efficiently because the detector is only exposed while it registers photons and because radiation is not emitted while the detector transfers the image signal to the computer [12].

The results of the CBCT devices expressed in Table 2 should be interpreted carefully due to the interplay among image quality, the size of the scanned volume, and the absorbed radiation dose in different tissues. Comparisons of the performances of CBCT devices cannot be done based on dosimetric results alone. The radiation dose from these devices can be seen as a function of the diagnostic application. The two key factors for an acceptable image are an appropriate size and positioning of the FOV and an acceptable quality of the reconstructed image [17], a point that was not evaluated in this revision. Further study is required to bring the image quality into play, on a technical and diagnostic level. By investigating technical image quality, the relation between the exposure from CBCT devices and the image quality performance can be quantified [17].

\section{Conclusions}

(1) Increases in $\mathrm{kV}, \mathrm{mA}$, exposure time, and FOV increase the dose of radiation, regardless of the type of exam.

(2) The effective dose for CT is greater than for CBCT or conventional radiographs.

(3) When the FMX is performed with round collimation, the ORD issues higher doses than most of the large FOV CBCT. Radiation dose for ORD can be lower than large FOV CBCT if rectangular collimation is used in FMX. Despite the image quality, CBCT does not replace the FMX and most orthodontic cases will be properly handled with conventional $2 \mathrm{D}$ radiographs. CBCT should be required for more complex cases.

(4) The orthodontists have the duty to preserve the health of the patient and always seek the best treatment. This quest begins with exams that require the least amount of radiation dose to treat the patient appropriately. 


\section{References}

[1] A. M. Bollen, J. Cunha-Cruz, and P. P. Hujoel, "Secular trends in preadult orthodontic care in the United States: 1942-2002," American Journal of Orthodontics and Dentofacial Orthopedics, vol. 132, no. 5, pp. 579-585, 2007.

[2] P. Hujoel, L. Hollender, A. M. Bollen, J. D. Young, M. McGee, and A. Grosso, "Head-and-neck organ doses from an episode of orthodontic care," American Journal of Orthodontics and Dentofacial Orthopedics, vol. 133, no. 2, pp. 210-217, 2008.

[3] E. D. M. M. Cerqueira, J. R. C. Meireles, M. A. Lopes et al., "Genotoxic effects of X-rays on keratinized mucosa cells during panoramic dental radiography," Dentomaxillofacial Radiology, vol. 37, no. 7, pp. 398-403, 2008.

[4] L. Hagmar, S. Bonassi, U. Strömberg et al., "Chromosomal aberrations in lymphocytes predict human cancer: a report from the European study group on cytogenetic biomarkers and health (ESCH)," Cancer Research, vol. 58, no. 18, pp. 4117 4121, 1998.

[5] A. Memon, S. Godward, D. Williams, I. Siddique, and K. AlSaleh, "Dental x-rays and the risk of thyroid cancer: a casecontrol study," Acta Oncologica, vol. 49, no. 4, pp. 447-453, 2010.

[6] J. M. Palomo, P. S. Rao, and M. G. Hans, "Influence of CBCT exposure conditions on radiation dose," Oral Surgery, Oral Medicine, Oral Pathology, Oral Radiology and Endodontology, vol. 105, no. 6, pp. 773-782, 2008.

[7] International Commission on Radiological Protection, “1990 recommendations of the international commission on radiological protection," Annals of the ICRP, vol. 21, no. 1-3, pp. 1-201, 1991.

[8] M. A. Garcia Silva, U. Wolf, F. Heinicke, K. Gründler, H. Visser, and E. Hirsch, "Effective dosages for recording Veraviewepocs dental panoramic images: analog film, digital, and panoramic scout for CBCT," Oral Surgery, Oral Medicine, Oral Pathology, Oral Radiology and Endodontology, vol. 106, no. 4, pp. 571577, 2008.

[9] National Council on Radiation Protection and Measurements, "Ionizing radiation exposure of the population of the United States," National Council on Raditation Protection Report number 160, National Council on Radiation Protection and Measurements, Bethesda, Md, USA, 2009.

[10] J. Valentin, "The 2007 recommendations of the International Commission on Radiological Protection. ICRP publication 103," Annals of the ICRP, vol. 37, no. 2-4, pp. 1-332, 2007.

[11] J. B. Ludlow and M. Ivanovic, "Comparative dosimetry of dental CBCT devices and 64-slice CT for oral and maxillofacial radiology," Oral Surgery, Oral Medicine, Oral Pathology, Oral Radiology and Endodontology, vol. 106, no. 1, pp. 106-114, 2008.

[12] J. B. Ludlow, L. E. Davies-Ludlow, S. L. Brooks, and W. B. Howerton, "Dosimetry of 3 CBCT devices for oral and maxillofacial radiology: CB Mercuray, NewTom 3G and i-CAT," Dentomaxillofacial Radiology, vol. 35, no. 4, pp. 219-226, 2006.

[13] J. B. Ludlow, L. E. Davies-Ludlow, and S. C. White, "Patient risk related to common dental radiographic examinations: the impact of 2007 International Commission on Radiological Protection recommendations regarding dose calculation," Journal of the American Dental Association, vol. 139, no. 9, pp. 1237-1243, 2008.

[14] W. De Vos, J. Casselman, and G. R. J. Swennen, "Cone-beam computerized tomography (CBCT) imaging of the oral and maxillofacial region: a systematic review of the literature,"
International Journal of Oral and Maxillofacial Surgery, vol. 38, no. 6, pp. 609-625, 2009.

[15] M. A. G. Silva, U. Wolf, F. Heinicke, A. Bumann, H. Visser, and E. Hirsch, "Cone-beam computed tomography for routine orthodontic treatment planning: a radiation dose evaluation," American Journal of Orthodontics and Dentofacial Orthopedics, vol. 133, no. 5, pp. 640.e1-640.e5, 2008.

[16] S. Gavala, C. Donta, K. Tsiklakis, A. Boziari, V. Kamenopoulou, and H. C. Stamatakis, "Radiation dose reduction in direct digital panoramic radiography," European Journal of Radiology, vol. 71, no. 1, pp. 42-48, 2009.

[17] R. Pauwels, J. Beinsberger, B. Collaert et al., "Effective dose range for dental cone beam computed tomography scanners," European Journal of Radiology, vol. 81, no. 2, pp. 267-271, 2012.

[18] D. C. Ngan, O. P. Kharbanda, J. P. Geenty, and M. A. Darendeliler, "Comparison of radiation levels from computed tomography and conventional dental radiographs," Australian Orthodontic Journal, vol. 19, no. 2, pp. 67-75, 2003.

[19] H. Visser, T. Rödig, and K. P. Hermann, "Dose reduction by direct-digital cephalometric radiography," Angle Orthodontist, vol. 71, no. 3, pp. 159-163, 2001.

[20] F. Gijbels, G. Sanderink, J. Wyatt, J. Van Dam, B. Nowak, and R. Jacobs, "Radiation doses of collimated vs non-collimated cephalometric exposures," Dentomaxillofacial Radiology, vol. 32, no. 2, pp. 128-133, 2003.

[21] F. Gijbels, G. Sanderink, J. Wyatt, J. Van Dam, B. Nowak, and R. Jacobs, "Radiation doses of indirect and direct digital cephalometric radiography," British Dental Journal, vol. 197, no. 3, pp. 149-152, 2004.

[22] K. Tsiklakis, C. Donta, S. Gavala, K. Karayianni, V. Kamenopoulou, and C. J. Hourdakis, "Dose reduction in maxillofacial imaging using low dose Cone Beam CT," European Journal of Radiology, vol. 56, no. 3, pp. 413-417, 2005.

[23] T. Okano, Y. Harata, Y. Sugihara et al., "Absorbed and effective doses from cone beam volumetric imaging for implant planning," Dentomaxillofacial Radiology, vol. 38, no. 2, pp. 79-85, 2009.

[24] A. Öhman, L. Kull, J. Andersson, and L. Flygare, "Radiation doses in examination of lower third molars with computed tomography and conventional radiography," Dentomaxillofacial Radiology, vol. 37, no. 8, pp. 445-452, 2008.

[25] R. A. Danforth and D. E. Clark, "Effective dose from radiation absorbed during a panoramic examination with a new generation machine," Oral Surgery, Oral Medicine, Oral Pathology, Oral Radiology, and Endodontics, vol. 89, no. 2, pp. 236243, 2000.

[26] J. K. Mah, R. A. Danforth, A. Bumann, and D. Hatcher, "Radiation absorbed in maxillofacial imaging with a new dental computed tomography device," Oral Surgery, Oral Medicine, Oral Pathology, Oral Radiology, and Endodontics, vol. 96, no. 4, pp. 508-513, 2003.

[27] J. A. Roberts, N. A. Drage, J. Davies, and D. W. Thomas, "Effective dose from cone beam CT examinations in dentistry," British Journal of Radiology, vol. 82, no. 973, pp. 35-40, 2009.

[28] M. Loubele, R. Bogaerts, E. Van Dijck et al., "Comparison between effective radiation dose of CBCT and MSCT scanners for dentomaxillofacial applications," European Journal of Radiology, vol. 71, no. 3, pp. 461-468, 2009.

[29] J. B. Ludlow, "A manufacturer's role in reducing the dose of cone beam computed tomography examinations: effect of beam filtration," Dentomaxillofacial Radiology, vol. 40, no. 2, pp. 115-122, 2011. 
[30] J. B. Ludlow, L. E. Davies-Ludlow, and S. L. Brooks, "Dosimetry of two extraoral direct digital imaging devices: newTom cone beam CT and Orthophos Plus DS panoramic unit," Dentomaxillofacial Radiology, vol. 32, no. 4, pp. 229-234, 2003.

[31] D. A. Miles and R. P. Langlais, "NCRP Report No. 145: new dental $\mathrm{x}$-ray guidelines: their potential impact on your dental practice," Dentistry Today, vol. 23, no. 9, pp. 128-134, 2004.

[32] American Dental Association Council on Scientific Affairs, "The use of dental radiographs: update and recommendations," The Journal of the American Dental Association, vol. 137, no. 9, pp. 1304-1312, 2006.

[33] V. Kumar, J. B. Ludlow, A. Mol, and L. Cevidanes, "Comparison of conventional and cone beam CT synthesized cephalograms," Dentomaxillofacial Radiology, vol. 36, no. 5, pp. 263 269, 2007.

[34] A. E. F. de Oliveira, L. H. S. Cevidanes, C. Phillips, A. Motta, B. Burke, and D. Tyndall, "Observer reliability of three-dimensional cephalometric landmark identification on cone-beam computerized tomography," Oral Surgery, Oral Medicine, Oral Pathology, Oral Radiology and Endodontology, vol. 107, no. 2, pp. 256-265, 2009.

[35] K. A. Misch, E. S. Yi, and D. P. Sarment, "Accuracy of cone beam computed tomography for periodontal defect measurements," Journal of Periodontology, vol. 77, no. 7, pp. 12611266, 2006.

[36] X. Liang, R. Jacobs, B. Hassan et al., "A comparative evaluation of cone beam computed tomography (CBCT) and multi-slice CT (MSCT) Part I. On subjective image quality," European Journal of Radiology, vol. 75, no. 2, pp. 265-269, 2010.

[37] N. Özmeric, I. Kostioutchenko, G. Hägler, M. Frentzen, and P. -M. Jervøe-Storm, "Cone-beam computed tomography in assessment of periodontal ligament space: in vitro study on artificial tooth model," Clinical Oral Investigations, vol. 12, no. 3, pp. 233-239, 2008.

[38] A. R. Lecomber, S. L. Downes, M. Mokhtari, and K. Faulkner, "Optimisation of patient doses in programmable dental panoramic radiography," Dentomaxillofacial Radiology, vol. 29, no. 2, pp. 107-112, 2000.

[39] A. R. Lecomber, Y. Yoneyama, D. J. Lovelock, T. Hosoi, and A. M. Adams, "Comparison of patient dose from imaging protocols for dental implant planning using conventional radiography and computed tomography," Dentomaxillofacial Radiology, vol. 30, no. 5, pp. 255-259, 2001.

[40] F. Gijbels, R. Jacobs, R. Bogaerts, D. Debaveye, S. Verlinden, and G. Sanderink, "Dosimetry of digital panoramic imaging. Part I: patient exposure," Dentomaxillofacial Radiology, vol. 34, no. 3, pp. 145-149, 2005.

[41] A. Suomalainen, T. Kiljunen, Y. Käser, J. Peltola, and M. Kortesniemi, "Dosimetry and image quality of four dental cone beam computed tomography scanners compared with multislice computed tomography scanners," Dentomaxillofacial Radiology, vol. 38, no. 6, pp. 367-378, 2009.

[42] X. M. Qu, G. Li, J. B. Ludlow, Z. Y. Zhang, and X. C. Ma, "Effective radiation dose of ProMax 3D cone-beam computerized tomography scanner with different dental protocols," Oral Surgery, Oral Medicine, Oral Pathology, Oral Radiology and Endodontology, vol. 110, no. 6, pp. 770-776, 2010.

[43] G. Scaf, A. G. Lurie, K. M. Mosier, M. L. Kantor, G. R. Ramsby, and M. L. Freedman, "Dosimetry and cost of imaging osseointegrated implants with film-based and computed tomography," Oral Surgery, Oral Medicine, Oral Pathology, Oral Radiology, and Endodontics, vol. 83, no. 1, pp. 41-48, 1997. 


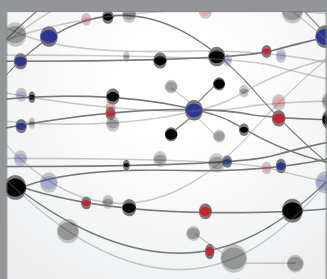

The Scientific World Journal
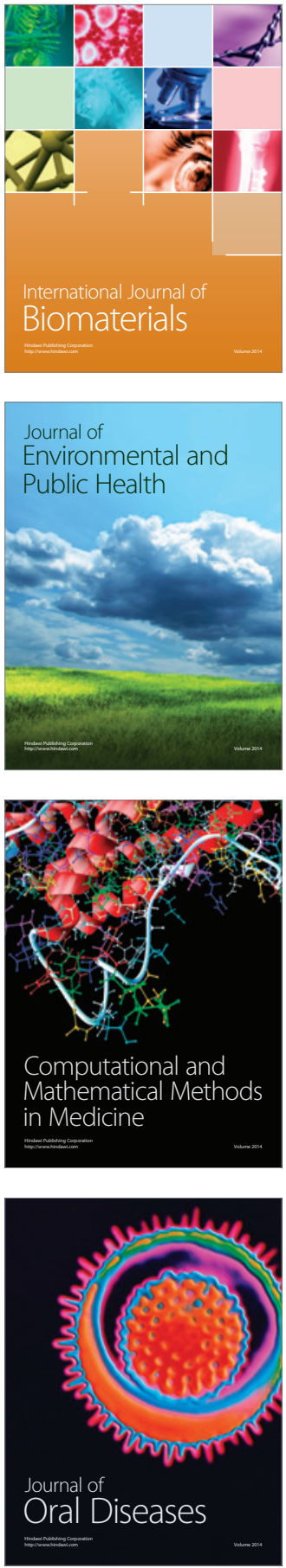
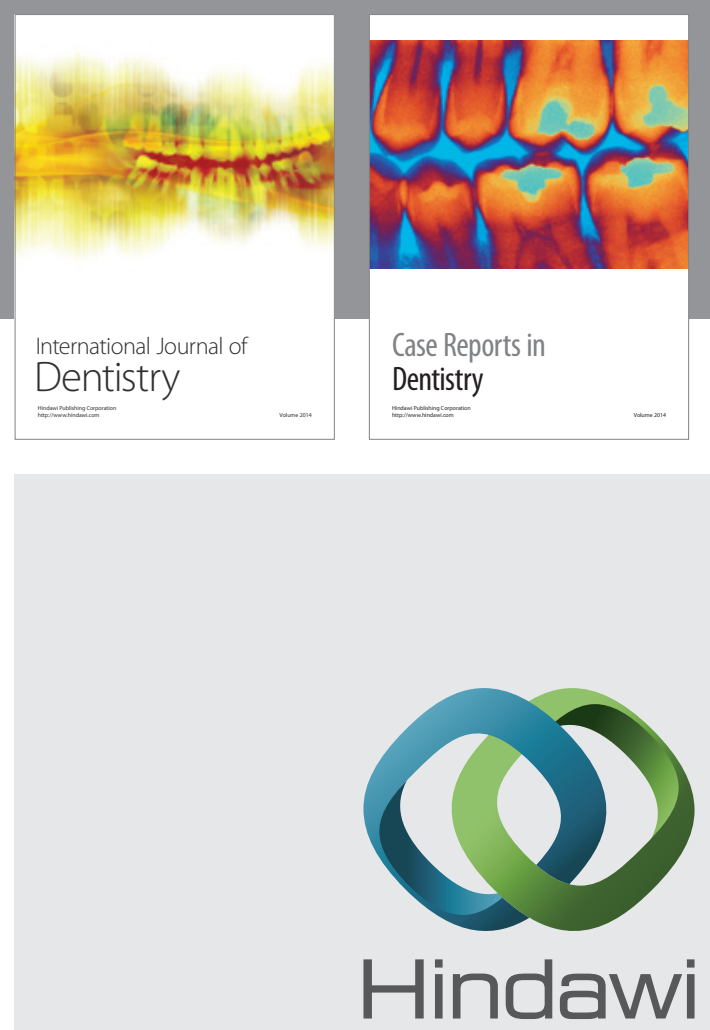

Submit your manuscripts at

http://www.hindawi.com
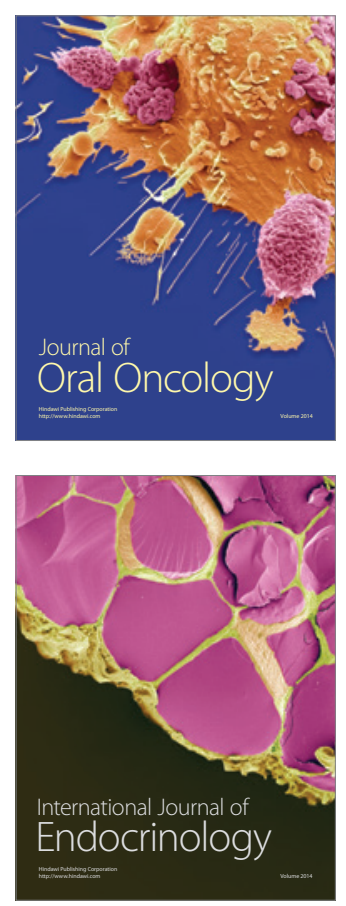
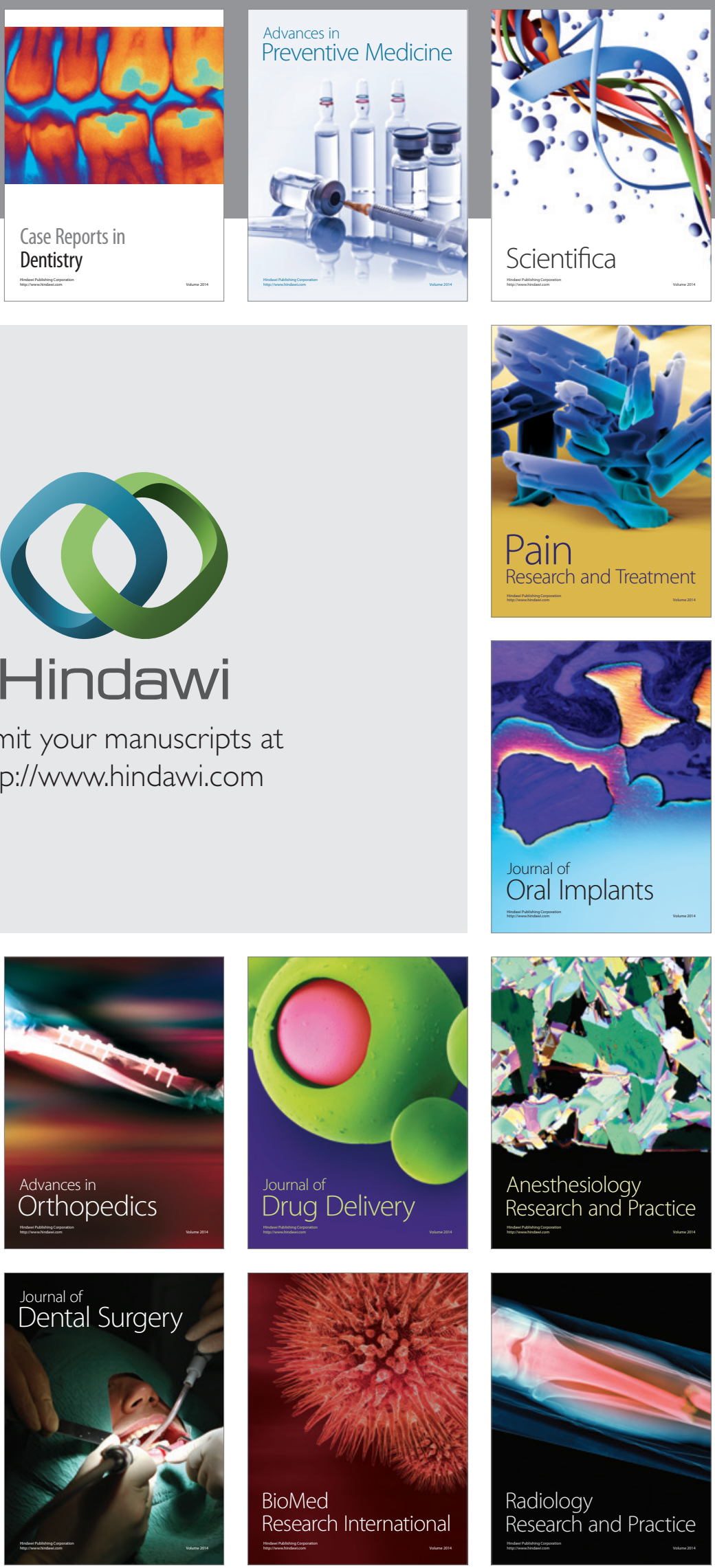